\title{
AR Status by Immunochemistry
}

National Cancer Institute

\section{Source}

National Cancer Institute. AR Status by Immunochemistry. NCI Thesaurus. Code C157165.

An indication that expression or absence of $A R$ in a sample was determined using immunohistochemical staining techniques. 